\title{
Contingência Semântica das Falas Materna e Paterna: Uma Análise Comparativa
}

\author{
Semantic Contingency in the Father's and Mother's Speech: A Comparative Analysis
}

\author{
Patrícia Nunes da Fonsêca* \& Nádia Maria Ribeiro Salomão \\ Universidade Federal da Paraíba
}

\begin{abstract}
Resumo
Este estudo teve como objetivo comparar a contingência semântica das falas paterna e materna, considerando a participação da criança na conversação. Participaram 12 crianças da classe média da cidade de João Pessoa/ $\mathrm{PB}$, com idade entre 24 a 31 meses, e seus respectivos genitores. Os genitores foram filmados em sua residência quando interagiam com suas crianças em uma situação de brincadeira livre por 20 minutos. Os resultados indicaram que as mães eram significativamente mais contínuas ao tópico da fala da criança do que os pais. Dentre os comportamentos contingentes, verificou-se que as mães expressaram mais reformulações e imitaram mais a fala dos meninos do que das meninas. Tais resultados foram discutidos considerando-se os contextos interativos e analisando-se as implicações das contingências da fala dos genitores para o processo de aquisição da linguagem.
\end{abstract}

Palavras-chave: Contingência; fala materna; fala paterna.

\begin{abstract}
The aim of this study is to compare the semantic contingency in the father's and mother's speeches directed to the child, considering the child's participation in the conversation. Twelve (12) children between 24 and 31 months old and their respective genitors of middle-class families from the city of João Pessoa, Pernambuco, Brazil, participated in this study. The parents were videotaped when interacting with their children in a free play situation for 20 minutes in their houses. The results indicated that the mothers gave significantly more continuity to the children's speeches when compared to the fathers. In relation to other contingent behaviour, the mothers presented more recast and imitations to the boys' speech than to the girls'. These results were discussed considering the level of the children's linguist development, the context in which the interactions occurred and analyzing the implications of contingency and breakdown speech to the process of language acquisition.
\end{abstract}

Keywords: Contingency, mother's speech, father's speech.

A literatura acerca da interação social e do desenvolvimento da comunicação na infância tem demonstrado um considerável crescimento, especialmente a partir de 1970, quando se incluiu a pragmática às pesquisas da linguagem (Borges \& Salomão, 2003).

Nesse novo cenário, a pragmática passa a ter um importante papel nas explicações acerca da aquisição e do desenvolvimento da linguagem, especialmente, nas pesquisas desenvolvidas pelos estudiosos que adotam a perspectiva da Interação Social (Braz \& Salomão, 2002; Conti-Ramsden, Hutcheson, \& Grove, 1995; Silva \& Salomão, 2002; Snow, 1997).

Conforme os pesquisadores da perspectiva da Interação Social, o contexto interativo é um espaço propício para o desenvolvimento das habilidades lingüísticas da criança, já que é nele que as trocas de experiências e de conhecimentos entre os interlocutores são efetivadas. Isso ocorre na medida em que a criança influencia o parceiro com seus comportamentos e suas características, ao mesmo tempo em que é influenciada pelos do outro, o que resulta numa relação recíproca e bidirecional.

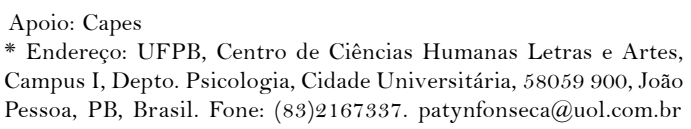

Os estudiosos da perspectiva da Interação Social consideram a criança como um ser ativo, desde o nascimento, e detentora de capacidades comunicativas que a fazem membro participante do contexto, entretanto, destacam o papel dos adultos na interação, em virtude destes serem mais experientes lingüisticamente e sensíveis às intenções comunicativas da criança, possibilitando assim, a criação de oportunidades para esta se desenvolver (Snow, 1997).

Para Pan, Imbens-Bailey, Winner, e Snow (1998) a interação diádica se estabelece quando os parceiros conversacionais compartilham a compreensão do tipo de atividade em que estão engajados, ou seja, as intenções de cada um na comunicação. Sob esse prisma, observa-se que os estudos acerca da aquisição da linguagem mostram que na interação adulto-criança, há um esforço do adulto tanto em compreender os sinais evocados pela criança, demonstrados por meio de gestos, balbucios e palavras, quanto em responder (Silva \& Salomão, 2002). Para tanto, os adultos apresentam modificações na fala dirigida à criança em comparação à fala dirigida a outros adultos. Essas modificações apresentam-se nos aspectos tonais, lexicais e sintáticos da linguagem, tornando uma fala simples, repetitiva, gramatical e semanticamente ajustada ao nível de compreensão da criança (Garton, 1992). 
Sokolov (1993), estudando as modificações na fala dos adultos dirigidas às crianças, verificou que as mudanças lingüísticas ocorriam tanto na fala materna quanto na paterna. Ademais, percebeu que os genitores acrescentavam ou apagavam certas classes de itens lexicais na fala dirigida às crianças. Ele observou que quanto mais "telegráfica” a fala da criança, maior a probabilidade dos pais e das mães usarem o item que estava faltando, seja um pronome ou/e substantivos. Por outro lado, este autor verificou que à medida que aumentava a competência lingüística da criança, os genitores tendiam a diminuir os acréscimos consideravelmente. Entretanto, os estudos (Conti-Ramsden et al., 1995; Tomasello, Conti-Ramsden, \& Ewert, 1990) mostram uma predominância dessas modificações na fala materna, devido a uma maior familiaridade da mãe com a fala da criança.

Conti-Ramsden (1994) comenta que a modificação da fala dos adultos ao nível da criança é favorável ao desenvolvimento lingüístico na medida em que essas mudanças permitem dar continuidade a conversação entre os parceiros conversacionais. Entretanto, Damast, TamisLeMonda, e Bornstein (1996) ressaltam que, não é simplesmente a continuidade do comportamento lingüístico da criança pela mãe que irá promover o desenvolvimento da linguagem, mas, sobretudo, a contingência e a adequabilidade do comportamento da mãe ao contexto de contínua interação.

Para Rice (1989), a contingência da fala consiste em uma combinação imediata do enunciado do adulto com o conteúdo ou tópico da fala da criança, onde se tem, como resultado, a continuidade da conversação.

Salomão (1996), estudando a interação mãe-criança, observou que as mães que responderam ao comportamento verbal da criança, dando continuidade ao seu tópico de interesse, promoveram oportunidades de maior participação no diálogo.

Analisando as falas materna e paterna dirigidas às crianças, Conti-Ramsden et al. (1995) verificaram que, de forma geral, as mães foram mais contingentes com a fala infantil do que os pais, o que permitia manter um tempo de conversa mais longo com a criança. Em contrapartida, os pais engajaram-se mais em episódios de interação nãoverbal. Conforme esses autores, isto pode ter ocorrido em decorrência do pouco conhecimento da fala infantil por parte dos pais que, por sua vez, ocasionou uma baixa no nível motivacional desses no que diz respeito a dar encadeamento ao tópico da fala das crianças.

Realizando estudo com as díades mãe/criança e pai/ criança, Tomasello et al. (1990) observaram que os enunciados não-contingentes dos adultos podem ser tão estimulantes para o desenvolvimento lingüístico infantil quanto os enunciados contingentes. Ademais, esses autores averiguaram que, na interação pai/criança havia muita "quebra" no diálogo e supuseram que este acontecimento poderia está relacionado ao fato dos pais não terem muita familiaridade com a linguagem infantil e, portanto, apresentarem uma certa dificuldade em compreender o que as crianças falavam. No entanto, para restabelecer o diálogo, os pais solicitavam da criança mais requisições de esclarecimentos dos enunciados infantis. Na medida em que isso ocorria, a criança deveria estar atenta ao discurso paterno e ao contex to interativo, para então, responder às requisições do pai de forma adequada.

Tomasello et al. (1990) sugerem a hipótese da "ponte". Segundo esta hipótese, o pai, por ser uma figura menos familiar, se comparado à mãe e, mais familiar do que as pessoas do contexto não-familiar, desempenha um papel intermediário entre a linguagem materna e a fala dos parceiros menos familiares. Isso ocorre porque o pai, ao se relacionar com a criança, exige lingüisticamente mais dela, se comparado à mãe. Dessa forma prepara a criança para se comunicar com pessoas que não têm nenhum conhecimento acerca das suas competências.

Dentre os comportamentos contingentes, pode-se encontrar a reformulação e a imitação. Snow (1989) afirma que a reformulação da fala infantil é um aspecto potencialmente facilitador do desenvolvimento lingüístico. Esta idéia é reiterada por Sokolov e Snow (1994), ao dizerem que a reformulação é um dos estilos de input mais eficazes no processo de aquisição da linguagem, em vista desse apresentar à criança versões da própria fala modificada, seja corrigida ou alternativa, isto é, dita com algumas palavras de forma diferente.

De acordo com a literatura (Conti-Ramsden, 1990; Snow, 1989; Sokolov \& Snow, 1994), a reformulação é um dos tipos de input lingüístico mais relevantes no processo de aquisição da linguagem. Isto se deve, sobretudo, à sua estrutura de fácil processamento, já que parte do enunciado apresentado pelo adulto consta no universo vocabular infantil. Ademais, Nelson, Welsch, Camarata, Butkovsky, e Camarata (1995) comentam que, devido à familiaridade com o vocabulário e com o tópico abordado na conversação, a reformulação tornase um input capaz de estimular e sustentar a atenção da criança.

Para Snow (1989), o sistema lingüístico da criança precisa ser instigado pelos enunciados que indiquem quais os aspectos da sua fala que devem estar errados e/ou insuficientes, pois, de outro modo, a criança não tem parâmetros para avaliar a sua linguagem, e assim, progredir lingüisticamente. Segundo essa autora, os enunciados dos genitores tornam-se adequados ao desenvolvimento lingüístico da criança, na medida em que eles são parcialmente ou totalmente compreensíveis, caso contrário, estas informações não lhes serão úteis.

O comportamento contingente pode também estar associado ao tipo de fala conhecida como feedback, porquanto ele é usado pelo adulto para confirmar, corrigir ou alterar um enunciado infantil, e nesse caso, repete parte ou toda a fala da criança. Essa repetição, para Hoff-Ginsberg, e Shatz (1982), é conhecida por expansão ou reformulação. Esse tipo de fala auxilia na aquisição lingüística por demonstrar à criança uma relação entre a linguagem primitiva e a linguagem apresentada pelo adulto, que pode ter sido corrigida e alternada, de modo a preencher parte de elementos lingüísticos omitidos nos enunciados. 
Nelson (1977) realizou um estudo com o objetivo de verificar a importância das reformulações dos enunciados das crianças. Para tanto, expôs um grupo de crianças a uma extensa quantidade de fala reformulada e comparou-o a outro grupo, que recebeu a mesma quantidade de conversa, porém sem reformulação. Diante dos dados, o autor concluiu que o grupo de crianças exposto à fala reformulada usou formas mais complexas em suas conversas do que as crianças do outro grupo.

No estudo de Conti-Ramsden (1990) houve uma comparação entre 28 díades mãe/criança, sendo 14 crianças com atraso na linguagem e 14 com desenvolvimento de linguagem normal. Em seu estudo, essa autora verificou que as crianças que apresentavam atraso na linguagem haviam recebido menos reformulações complexas da mãe do que as crianças com desenvolvimento lingüístico normal. Em vista disto, concluiu que a relativa ausência de reformulações no input materno pode ser uma das explicações para o atraso da fala das crianças.

Apesar dos vários estudos demonstrarem a relevância das reformulações (Hoff-Ginsberg \& Shatz, 1982; Nelson et al., 1995; Snow, 1989; Conti-Ramsden, 1990; Sokolov \& Snow, 1994) observa-se que, se esse tipo de fala não for apresentado de modo desafiante, de modo que possa estimular a atenção e a participação da criança, não haverá efeito favorável, mesmo que sua freqüência mostre-se elevada.

Esse fato, segundo Nelson et al. (1995), pode ser explicado a partir da "teoria da aprendizagem do efeito raro", Rare Event Learning - REL. De acordo com essa teoria, a criança tem habilidades para processar, de forma eficiente, a linguagem apresentada pelos adultos e, a partir de então, aprender estruturas lingüísticas. No entanto, esta linguagem, segundo os esses autores, deve ser apresentada em um nível um pouco mais elevado do que o nível lingüístico da criança, mas passível de ser processada por ela, caso contrário ela não terá o efeito esperado. Nelson et al. (1995) concluem que as reformulações e a continuação do tópico de interesse da criança mostram-se como estruturas importantes no desenvolvimento de sua linguagem.

A imitação é um outro tipo de fala contingente que, de acordo com Demetras, Post, e Snow (1986), possibilita à criança ter indicadores acerca do seu enunciado, pois, geralmente, a mãe que repete, na íntegra, o enunciado infantil sinaliza para criança que o seu enunciado está correto, isto é, dá um feedback.

Fagot e Hagan (1991), estudando os feedbacks maternos, verificaram que esse estilo de fala é encorajador do potencial comunicativo da criança, pois além de possibilitar-lhe a reinterpretação da fala anteriormente proferida, ainda permite manter a criança na conversação.

Comparando o comportamento comunicativo dos pais e das mães dirigidos às crianças durante um contexto interativo Conti-Ramsden et al. (1995) observaram que as mães participavam mais ativamente da interação e imitavam significativamente mais a fala infantil do que os pais.
Contudo, analisar a linguagem num contexto sóciocomunicativo implica em investigar os aspectos desse contexto que são favoráveis ou desfavoráveis à promoção das habilidades comunicativas. Dentre esses aspectos, encontram-se as falas materna e paterna, o contexto interativo e as intenções dos falantes na interação. Desse modo, este estudo tem por objetivo comparar a contingência semântica das falas maternas e paternas no contexto de brincadeira livre em ambiente familiar, considerando o gênero das crianças e a participação destas na interação.

\section{Método}

\section{Participantes}

Participaram desse estudo 24 díades, sendo 12 díades mãecriança e 12 díades pai-criança. As famílias pertenciam à classe média da cidade de João Pessoa/PB. A faixa etária dos pais ficou entre 26 e 42 anos $(m=34, d p=4,71)$ e das mães variou entre 25 e 39 anos $(m=30, d p=4,76)$. Com relação ao nível de escolaridade, verificou-se que, no grupo dos pais, oito participantes apresentaram o ensino superior completo e os demais o ensino superior. No grupo das mães, nove delas tinham ensino superior completo e apenas 4 tinham o ensino médio. As crianças eram de ambos os sexos, sendo 6 meninos e 6 meninas, com idades variando entre 24 a 31 meses.

\section{Instrumentos}

Os instrumentos utilizados neste estudo consistiram de: - uma câmara de vídeo para gravar as observações das interações das díades.

- um vídeo-cassete para transcrever as gravações das observações.

\section{Procedimentos}

O contato inicial com os genitores das crianças se deu por telefone, onde foram dadas algumas explicações acerca da pesquisa e agendada uma visita à residência dos participantes. Na primeira visita foi entregue um documento, o qual solicitava a participação do casal no estudo e a permissão para o uso da câmera de vídeo, bem como assegurava o sigilo das informações coletadas. Nesse momento, foram agendadas as visitas para a realização das filmagens.

As filmagens foram realizadas nas residências dos participantes e ocorreram em momentos distintos, sempre com uma mudança na ordem das díades para contrabalançar o seu efeito nos resultados. As interações aconteceram em uma situação de brincadeira livre, em vista desta constituirse um exemplo das práticas de socialização típicas do cotidiano familiar e, ainda, por possibilitar maior liberdade aos genitores quanto à forma de se adaptarem ao nível de compreensão da criança durante a brincadeira. Cada sessão de filmagem teve a duração de 20 minutos, e antes de cada uma delas, era dada a seguinte instrução aos genitores: "mamãe/papai brinque com sua criança da maneira que costumeiramente brinca”. 


\section{Análise dos dados}

Para a análise das observações foram utilizados os 10 minutos intermediários de cada filmagem. A análise foi realizada em quatro etapas: a primeira consistiu em transcrever as observações das interações, segundo as normas do CHAT (Codes for Human Analysis of Transcripts), componente do programa computacional CHILDES (Child Language Data Exchange System). Na segunda etapa, realizouse uma leitura minuciosa dos protocolos, o que permitiu a elaboração de um conjunto de categorias acerca da contingência semântica da fala dos genitores e da participação da criança. Na terceira, obteve-se a freqüência dos enunciados dos genitores e das crianças, através do CLAN (Computerised Language Analysis). Por fim, na quarta etapa, a pesquisadora inseriu os dados no pacote estatístico SPSS (Statistical Package for the Social Sciences), e realizou o teste de significância não-paramétrico Mann-Whitney.

A contingência semântica da fala dos genitores foi analisada considerando as seguintes categorias: Enunciados de Continuação-dizem respeito aos enunciados, posteriores à fala prévia da criança, que dão continuidade à verbalização infantil, uma vez que abordam o mesmo conteúdo semântico presente nesta. Esses enunciados não se apresentam como resultados de reformulações ou imitações da fala infantil. Enunciados de Não-Continuação - são os enunciados que não dão continuidade à verbalização infantil, em vista dos genitores introduzirem um novo conteúdo semântico à conversação. Reformulação - trata-se de enunciados, que dão continuidade à verbalização infantil, uma vez que se referem ao mesmo conteúdo semântico exposto pela criança. Entretanto, esses enunciados caracterizam-se por apresentarem mudanças estruturais da verbalização precedente da criança. Imitações - são enunciados que dão continuidade à verbalização infantil, uma vez que se referem ao mesmo conteúdo semântico exposto pela criança. Esses enunciados, entretanto, caracterizam-se por se apresentarem como resultado de uma imitação integral da verbalização infantil. Outros - são enunciados que se apresentam posteriores à fala ininteligível da criança.

A participação da criança foi analisada considerando as seguintes categorias: Fala espontânea - enunciado que não é precedido por uma questão, um pedido, ou uma ordem dos genitores nem é o resultado de uma imitação das verbalizações prévias destes. Resposta Verbal e Não-Verbal Adequada - comportamento verbal e não-verbal que responde, de modo adequado, ao enunciado prévio dos genitores. Resposta Verbal e Não-Verbal Inadequadacomportamento verbal e não-verbal que responde, de modo inadequado, ao enunciado prévio dos genitores. Fala Ininteligível - enunciado em que se torna impossível compreender o que a criança está dizendo. Comportamento Não-Verbal Espontâneo - comportamento apresentado pela criança, independente de uma requisição, ordem, questão ou uma ação prévia dos genitores. Comportamento Não-Verbal Contínuo - diz respeito ao comportamento apresentado pela criança, que tem por função dar continuidade à ação prévia dos genitores. Nessa categoria, não foram considerados os comportamentos frutos de imitação da ação dos genitores.

\section{Resultados}

A análise do sistema de categorias da contingência semântica da fala dos genitores foi realizada em quatro fases: na primeira, compararam-se os dados dos pais e das mães, independentemente do gênero das crianças; na segunda, confrontou-se a contingência da fala dos genitores dos meninos com a contingência da fala dos genitores das meninas; na terceira fase, foram comparados os dados da contingência semântica dos pais com os das mães dos meninos, finalizando-se, então com a comparação dos dados dos pais com os das mães das meninas.

A Tabela 1 demonstra que houve diferenças significativas entre as médias de postos das categorias continuidade e imitação, cujos $p<0,05$. Esses resultados mostraram que as mães utilizaram mais enunciados que dão continuidade ao tópico da fala das crianças do que os pais, do mesmo modo que imitaram mais os enunciados infantis do que os pais.

Conforme a Tabela 2, verifica-se que houve diferenças significativas entre as médias de postos da categoria reformulação. Isto implica dizer que os pais e as mães dos meninos apresentaram um número maior de enunciados reformulados da fala das crianças, quando comparados aos enunciados evocados pelos genitores das meninas.

Através da Tabela 3, pode-se notar que houve diferença significativa nas médias de postos das falas dos pais e das mães dos meninos, na categoria continuidade. Esse resultado indicou que as mães foram significativamente mais contínuas ao tópico do enunciado das crianças do que os pais, o que vem reforçar as conclusões da primeira comparação realizada neste estudo, acerca da contingência semântica da fala dos genitores.

A Tabela 4 exibe a diferença significativa entre as médias de postos da contingência da fala dos pais e das mães das meninas na categoria continuidade. Este resultado corrobora com o que se constatou na comparação do sistema de contingência entre os genitores das crianças (meninos e meninas) e na comparação dos genitores dos meninos, ou seja, que as mães utilizaram mais enunciados de continuidade da fala das crianças do que os pais.

Para se ter uma compreensão mais ampla sobre os resultados da contingência da fala paterna e da materna, faz-se necessário averiguar a participação da criança, tendo em vista a importância que esta apresenta no estabelecimento do processo interativo.

Para análise da participação da criança, considerou-se a freqüência dos enunciados infantis segundo as categorias elaboradas e aplicou-se o teste estatístico Mann-Whitney aos resultados para verificar o nível de significância.

Na comparação da participação dos meninos com as mães e com os pais, percebeu-se que estes apresentaram diferenças significativas quanto à fala ininteligível. Este resultado mostrou que os meninos falaram mais de forma incompreensível com os pais do que com as mães.

Com relação às meninas, observou-se que, na interação com as mães, estas apresentaram diferenças significativas no comportamento não-verbal contínuo. Nesse caso, não 
Fonseca, P.N. \& Salomão, N.M.R. (2006). Contingência Semântica das Falas Maternas e Paternas: Uma Análise Comparativa.

Tabela 1

Comparação da Contingência Semântica da Fala Paterna e da Fala Materna

\begin{tabular}{lcccc}
\hline Contingência Semântica & Pais & Mães & \multicolumn{2}{c}{ Teste de Mann- Whitnney } \\
\cline { 2 - 5 } & Meninos e Meninas & Meninos e Meninas & & \\
& Média de Postos & Média de Postos & Estatística U & Estatística p \\
\hline Não continuidade & 14,17 & 10,83 & 52,00 & 0,266 \\
Continuidade & 8,83 & 16,17 & 28,00 & $0,01^{*}$ \\
Reformulação & 9,88 & 15,13 & 40,50 & 0,068 \\
Imitação & 9,46 & 15,54 & 35,50 & $0,033^{*}$ \\
Outros & 14,04 & 10,96 & 53,50 & 0,291 \\
\hline
\end{tabular}

Nota. ${ }^{*} p<0,05$ (valores significativos)

Tabela 2

Comparação da Contingência Semântica da Fala dos Genitores segundo o Gênero das Crianças

\begin{tabular}{lcccc}
\hline Contingência Semântica & \multicolumn{2}{c}{ Pais } & Meste de Mann- Whitnney \\
\cline { 2 - 5 } & $\begin{array}{c}\text { Meninos e Meninas } \\
\text { Média de Postos }\end{array}$ & $\begin{array}{c}\text { Meninos e Meninas } \\
\text { Média de Postos }\end{array}$ & Estatística U & Estatística p \\
\hline Não continuidade & 13,42 & 11,58 & 61,00 & 0,551 \\
Continuidade & 13,38 & 11,63 & 61,50 & 0,551 \\
Reformulação & 15,38 & 9,63 & 37,50 & $0,045^{*}$ \\
Imitação & 13,04 & 11,96 & 65,50 & $0,713^{*}$ \\
Outros & 13,46 & 11,54 & 60,50 & 0,514 \\
\hline Nota. ${ }^{*} p<0,05$ (valores significativos) & & & &
\end{tabular}

Tabela 9

Comparação da Contingência Semântica da Fala dos Genitores dos Meninos

\begin{tabular}{lcccc}
\hline Contingência Semântica & \multicolumn{2}{c}{ Pais } & Mães & Teste de Mann- Whitnney \\
\cline { 2 - 5 } & $\begin{array}{c}\text { Meninos e Meninas } \\
\text { Média de Postos }\end{array}$ & $\begin{array}{c}\text { Meninos e Meninas } \\
\text { Média de Postos }\end{array}$ & Estatística U & Estatísticap \\
\hline Não continuidade & 7,17 & 5,83 & 14,00 & 0,589 \\
Continuidade & 4,33 & 8,67 & 5,00 & $0,041^{*}$ \\
Reformulação & 4,67 & 8,33 & 7,00 & 0,093 \\
Imitação & 4,58 & 8,42 & 6,50 & 0,065 \\
Outros & 8,42 & 4,58 & 6,50 & 0,065 \\
\hline Nota
\end{tabular}

Nota. $* p<0,05$ (valores significativos)

Tabela 4

Comparação da Contingência Semântica da Fala dos Genitores das Meninas

\begin{tabular}{lcccc}
\hline Contingência Semântica & Pais & Mães & Teste de Mann-Whitnney \\
\cline { 2 - 5 } & $\begin{array}{c}\text { Meninos e Meninas } \\
\text { Média de Postos }\end{array}$ & $\begin{array}{c}\text { Meninos e Meninas } \\
\text { Média de Postos }\end{array}$ & Estatística U & Estatística p \\
\hline Não continuidade & 7,17 & 5,83 & 14,00 & 0,589 \\
Continuidade & 4,17 & 8,83 & 4,00 & $0,026^{*}$ \\
Reformulação & 5,00 & 8,00 & 9,00 & 0,180 \\
Imitação & 5,50 & 7,50 & 12,00 & 0,394 \\
Outros & 6,42 & 6,58 & 17,50 & 0,937 \\
\hline
\end{tabular}

Nota. ${ }^{*} p<0,05$ (valores significativos)

foram considerados os comportamentos frutos de imitação da ação dos genitores. Quanto à interação pai-criança não foram verificadas diferenças significativas.

\section{Discussão e Conclusão}

A discussão é apresentada conforme as categorias da contingência semântica da fala dos genitores e, posteriormente, se faz uma análise da participação das crianças no contexto interativo.

Quanto à categoria continuidade, os resultados mostraram que as mães apresentaram significativamente mais enunciados de continuidade da fala infantil do que os pais, aspecto verificado também quando se comparou o pai e a mãe segundo o gênero da criança. Esse resultado pode ser explicado a partir dos estudos de Tomasello et al. (1990), 
Conti-Ramsden et al. (1995) e Snow (1997), os quais afirmam que pelo fato das mães conhecerem melhor as capacidades comunicativas da criança, elas têm mais facilidade de se envolverem, de modo predominante, nos mais variados lugares e nas mais diversas atividades com a criança, o que as possibilita ajustar a sua fala aos enunciados infantis, modo que a criança as compreenda. Esse ajuste lingüístico, de acordo com os autores citados, ocorre em termos sintáticos, semânticos e fonéticos, e depende do nível cognitivo e lingüístico da criança.

Em relação à categoria reformulação, os resultados da comparação dos genitores em função do gênero das crianças indicam que o pai e a mãe dos meninos apresentaram significativamente mais enunciados reformulados do que os genitores das meninas. Tais resultados revelaram a atenção dos genitores dos meninos em reformular os enunciados da criança, principalmente, aqueles que se apresentaram de forma incorreta, ou seja, com um erro de ordem fonética, sintática ou semântica. Com essa atitude de corrigir, reformulando os enunciados infantis, esses pais estão sinalizando para a criança onde está o erro, aspecto que, segundo Snow (1989), é extremamente importante para o curso do desenvolvimento da linguagem.

A partir de uma leitura exaustiva dos protocolos, observouse que as reformulações das falas materna e paterna tinham, em geral, a função de reformular os enunciados infantis quando emitiam feedbacks à criança, especialmente o feedback de confirmação, onde o adulto reformula o enunciado da criança a fim de confirmar-lhe as verbalizações, embora demonstre tê-las entendido. Essas respostas normalmente se apresentam de forma interrogativa, o que instiga uma resposta da criança e, portanto, permite a continuidade da interação, como pode ser visto no exemplo a seguir: criança: A tartaruguinha aqui. pai: É uma tartaruguinha, é? criança: É a tartaruguinha.

No que se refere aos resultados da categoria imitação, verificou-se que as mães apresentaram significativamente mais esta categoria, quando comparadas aos pais. Fazendo uma leitura exaustiva dos protocolos, observou-se que as imitações apresentadas pelas mães se deram em forma de feedback, especialmente de feedback de aprovação, o que demonstra para criança que o seu enunciado está correto, ao mesmo tempo em que incita à continuidade na conversação. Segundo Bohannon, e Stanowicz (1988) esse tipo de comportamento materno possibilita à criança reinterpretar sua fala anteriormente proferida e assim desenvolver a linguagem mais rapidamente do que quando a mãe apresenta a imitação em menor quantidade.

$\mathrm{Na}$ categoria não-continuidade, não se observaram diferenças significativas, embora seja interessante chamar a atenção para os valores das médias de postos dos pais e das mães referentes a esta categoria. Em todas as comparações realizadas, percebeu-se que os pais sempre obtiveram médias de postos superiores às médias das mães, o que sugere a não-continuidade dos enunciados infantis por parte dos pais.
A descontinuidade do discurso infantil na interação pai/ criança foi observada em estudo desenvolvido por Tomasello et al. (1990), que observou uma exigência maior acerca da linguagem infantil por parte do pai do que da mãe e, portanto, um maior número de "quebra" no diálogo pai/ criança. Para estes autores, a não-continuidade permitia a criança observar, mais atentamente, a fala e as ações paternas presentes no contexto interativo, o que se tornaria um aspecto importante para o desenvolvimento da linguagem.

Tomasello et al. (1990) ainda ressalta que, pelo fato do pai emitir uma fala que se apresenta em um nível um pouco acima do nível lingüístico infantil, este instigaria a curiosidade e a participação da criança no diálogo, ao mesmo tempo em que promoveria o seu avanço lingüístico.

Com relação à participação da criança, os resultados levam a supor que os meninos participaram mais ativamente do contexto interativo do que as meninas. Isto pode estar relacionado ao fato deles terem sido bastante solicitados pelos genitores a darem explicações dos seus enunciados, já que os mesmos demonstraram um número elevado de inelegibilidade na fala. Esse fato vem mostrar a importância de se fazer uma análise bidirecional, onde se considera o comportamento de ambos os participantes da interação.

Revisando todo o sistema de categorias do sistema de contingências dos genitores, pode-se verificar que o pai diferiu da mãe, em alguns aspectos, na forma de dar encaminhamento aos enunciados da criança, cada um com um estilo próprio, dentro de suas características individuais e de seus papéis sociais.

Sendo assim, pode-se concluir que, assim como a mãe, o pai é um personagem importante no desenvolvimento infantil, de modo particular, na área lingüística. Dentro desse contexto, Rondal (1980) afirma que há uma complementariedade do papel dos genitores no desenvolvimento infantil, porquanto eles promovem situações e conhecimentos distintos.

Por fim, destaca-se a importância de novas investigações que abordem o estudo das díades mãe/criança e pai/criança em outros contextos e das tríades mãe/criança/pai. Esses estudos poderão permitir a observação de possíveis semelhantes e diferenças nos padrões de comportamento dos genitores e a influência destes no desenvolvimento da linguagem infantil. Ademais, é pertinente estudar a interação da criança com outras pessoas que participam do seu dia-a-dia, como por exemplo: as avós e as babás.

\section{Referências}

Borges, L.C. \& Salomão, N.M.R. (2003). Aquisição da linguagem: Considerações da perspectiva da Interação Social. Psicologia: Reflexão छ Crítica, 16, 327-336.

Bohannon, J.N. \& Stanowicz, I. (1988). The issue of negative evidence: adult responses to children's language errors. Development Psychology, 24, 684-689.

Braz, F.S. \& Salomão, N.M.R. (2002). A fala dirigida a meninos e meninas: um estudo sobre o input materno e suas variações. Psicologia: Reflexão \& Crítica, 15, 333-344. 
Conti-Ramsden, G. (1990). Maternal recasts and other contingent replies to language-impaired children. Journal of Speech and Hearing Disorders, 55, 262-274.

Conti-Ramsden, G. (1994). Language interaction with atypical language learners. In C. Gallaway \& B. Richards (Eds.), Input and interaction in language acquisiton (pp.183-198). London: Cambridge University Press.

Conti-Ramsden, G. Hutcheson, G.D., \& Grove, J. (1995). Contingency and breakdown: children with SLI and their conversations with mothers and fathers. Journal of Speech and Hearing Research, 38, 1290-1302.

Damast, A.M., Tamis-LeMonda, C.S., \& Bornstein, M.H. (1996). Mother-child play: sequential interactions and the relation between maternal beliefs and behaviors. Child Development, 67, 1752-1766.

Demetras, M.J., Post, K.N., \& Snow, C.E. (1986). Feedback to first language learners: the role of repetitions and clarification questions. Journal of Child Language, 13, 275-292.

Fagot, B.A. \& Hagan, R. (1991). Observations of parental reactions to sex-stereotyped behaviors: age and sex effects. Child Development, 62, 617-628.

Garton, A.F. (1992). Social interaction and the development of language and cognition. Hillsdale: Lawrence Erlbaum.

Hoff-Ginsberg, E., \& Schatz, M. (1982). Linguistic input and the child's acquisition of language. Psychological Bulletin, 92, 3-26.

Nelson, K.E. (1977). Facilitating children's syntax acquisition. Developmental Psychology, 13, 101-107.

Nelson, K.E., Welsch, J., Camarata, S.M., Butkovsky, L., \& Camarata, M. (1995). Available input for language impaired children and younger children of matched language levels. First Language, 15, 1-17.

Pan, B.A., Imbens-Bailey, A., Winner, K., \& Snow, C. (1998). Communicative intents expressed by parents in interaction with young children. Harvard University Graduate School of Education.

Rice, M.L. (1989). Children's language acquisition. American Psychologist, 44, 149-156.

Rondal, J.A. (1980). Fathers' and mother' speech in early language development. Journal Child Language, 7, 353-369.

Salomão, N.M.R. (1996). Interaction between mothers and children with specific language impairment; A longitudinal study. Tese de Doutorado não-publicada, University of Manchester. Manchester, England.

Salomão, N.M.R., \& Conti-Ramsden, G. (1994). Maternal speech to their offspring: SLI children and their younger siblings. Scandinavian Journal of Logopedios and Phonology, 19, 11-17.
Silva, M.P.V. \& Salomão, N.M.R. (2002). Interações verbais e não-verbais entre mães-crianças portadoras de Síndrome de Down e entre mães-crianças com desenvolvimento normal. Estudos de Psicologia, 7, 311-323.

Snow, C.E. (1997). Questões no estudo do Input: sintonia, universalidade, diferenças individuais e evolutivas, e causas necessárias. In P. Fletcher \& B. Mac Whinney (Eds.), Compêndio de linguagem da criança (pp.153-163). Porto Alegre: ArtMed.

Snow, C.E. (1989). Understanding social interaction and language acquisition: sentences are not enough. In M.H. Bornstein \& J.S. Bruner (Eds.), Interaction in Human Development (pp.83-99). Hillsdale, NJ: Lawrence Erlbaum.

Sokolov, J.L. (1993). A local contingency analysis of the finetuning hypothesis. Developmental Psychology, 29,1008-1023.

Sokolov, J.L., \& Snow, C.E. (1994). The changing role of negative evidence in theories of language development. In C. Gallaway $\&$ B. Richards (Eds.), Input and interaction in language acquisiton (pp.38-55). London: Cambridge University Press.

Tomasello, M., Conti-Ramsden, G., \& Ewert, B. (1990). Young children's conversations with their mothers and fathers: Differences in breakdown and repair. Child Language, 17, $115-$ 130

Sumissão: 25/08/2004 $1^{a}$ revisão: 26/11/2004 Última revisão: 08/03/2005 Aceite final: 19/04/2005 